Prog. Neuro-Psychopharmacol. Vol.4, pp.309-312.

Pergamon Press Ltd, 1980. Printed in Great Britain.

\title{
STRESS INDUCED FACIITTATION OF OPIATE CATALEPSY IN THE RAT
}

\author{
Richard J. Katz \\ Mental Health Research Institute, Departinent of Psychiatry \\ The University of Michigan Medical Center \\ Ann Arbor, MI \\ (Final form, May 1980)
}

\begin{abstract}
1. Adult male Sprague-Dawley rats were injected with a standard low dose of opiate (10 mg/kg of morphine sulfate).

2. Experimental subjects received one of two stressful procedures (tail pinch, floor cooling) while control subjects were not disturbed.

3. Using motor activity as an index of catalepsy, both stressful procedures prolonged the syndrome.

4. Stress may therefore affect this opiate mediated process.
\end{abstract}

Key words: activity, catalepsy, morphine, opiate, stress, tail pinch, tonic immobility

\section{Introduction}

When rats are injected with moderate to high doses of opiates they cease moving and become rigid. This response has been termed catalepsy although it is behaviorally, anatomically, and psychopharmacologically distinct in comparison with neuroleptic catalepsy, and in fact catatonia may be a more accurate description (Costall and Naylor, 1974; Nakamura et al, 1973). The cataleptic state (because catalepsy appears to have greater current acceptance it will be used to indicate the behavioral state in question) was one of the earliest reported effects of opiates in rats and early and subsequent observations have been summarized elsewhere (Krueger et al, 1941). From these and more recent reports it is clear that the syndrome is not a generalized motor deficit. When drug treated rats are subjected to various forms of environmental stimulation, they initiate motor activity, although it is not necessarily sustained (Krueger et al, 1941). Few formal or quantified descriptions of this last phenomenon are available and it is not known how more prolonged stress might affect behavior. Observations to date indicate catalepsy involves both a loss of motor initiation and also of sustained activity, however, and they may be viewed as dissociable functions. Both to replicate earlier less quantified reports and to extend the behavioral characterization of a morphine effect we examined rats after a moderate dose of morphine, with and without two environmental manipulations. Our findings support the proposed behavioral fractionation of the opiate syndrome into distinctive classes of alterations.

\section{Methods}

Subjects: Twenty four adult male Sprague-Dawley rats 250-300 g each were maintained, six rats per cage with food (Teklad 4.08 fat rodent diet S-0836; Madison, WI) and tap water continuously available. Twelve hour light/dark cycles (lights on $=8: 00-20: 00 \mathrm{~h}$ ) were automatically programmed. 
Apparatus and Procedures: Details of apparatus and recording procedure have been published elsewhere (e.g. Katz and Carroll, 1978). Briefly, the apparatus consisted of four commercially available (Stoelting, Chicago) tuned oscillators operating on a selective mode for the detection of gross body movement. All sensing of movement was remote and unobtrusive. Rats were individually placed in $48 \times 27 \times 20 \mathrm{~cm}$ polypropylene cages with fresh pine chip bedding. The cages were then placed upon the activity monitors for recording. The first $60 \mathrm{~min}$ served as a habituation period. Rats were then removed and injected with $10 \mathrm{mg} / \mathrm{kg}$ of morphine sulfate in a $0.9 \%$ sodium chloride vehicle. Injections utilized a $5 \mathrm{mg} / \mathrm{cc}$ solution. Twelve subjects served as controls, and remained undisturbed.

Two experimental groups of six rats each were given identical treatment, but were also subjected to one of two environmental manipulations. The first experimental group was subjected to a mild chronic tail pinch by a pair of rubber dam forceps closed to the first notch and placed $3 \mathrm{~cm}$ from the base of the tail. The second experimental group was tested in a box the floor of which was chronically lowered to $4 \mathrm{C}$ by an ice bath. Both manipulations were carried out ten to twenty minutes after drug injection and the experimental manipulations continued until the conclusion of testing 90 minutes later.

Statistical Analysis: Motor activity was recorded throughout the session at ten minute intervals. All data are presented as means and standard errors, and were analyzed using repeated measures analysis of variance (Bruning and Kintz, 1977).

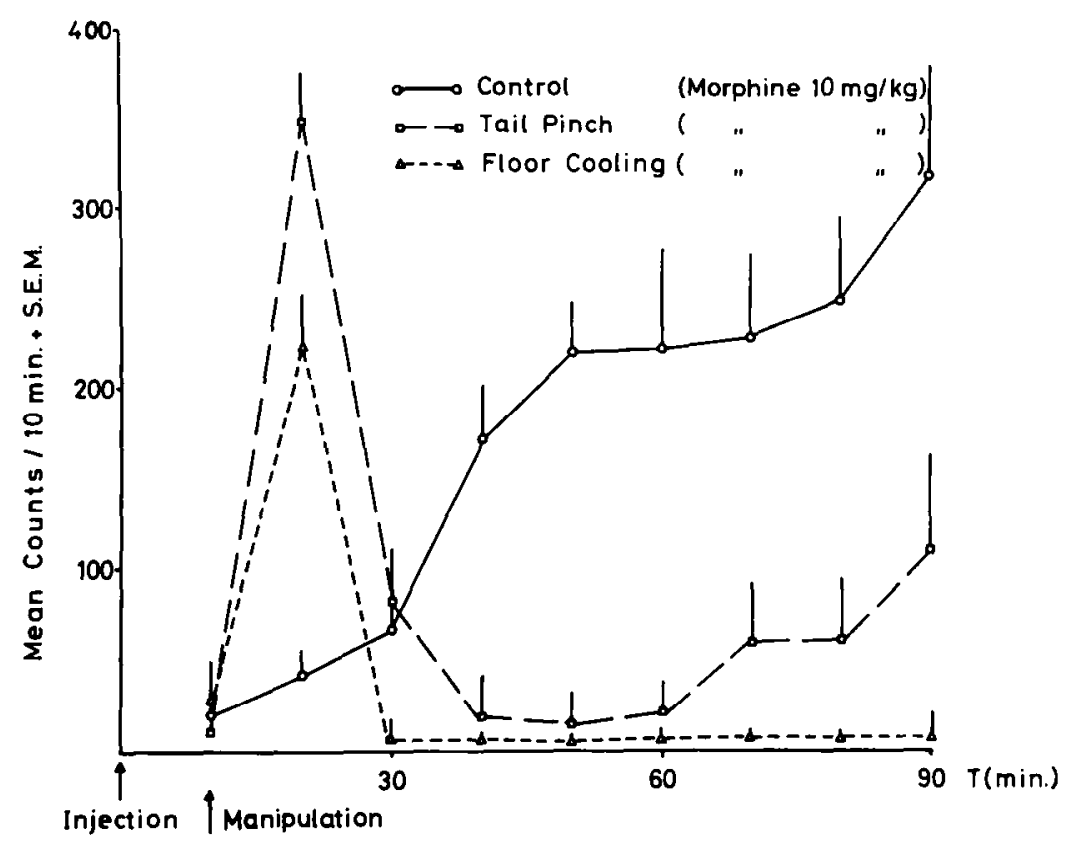

Fig. 1. Effects of tail pinch (TP) and floor cooling (FC) upon opiate immobility induced by morphine $(10 \mathrm{mg} / \mathrm{kg})$ (CTC = control). Both experimental manipulations begin between min 10-20. All values = mean and standard error. 


\section{Results}

The experimental subjects showed significantly greater immobility based upon remote motor recording. In Figure One it may be seen that all drug treated subjects were initially immobile. The experimental manipulations induced an initial increase in motor activity (min 10-20) and a subsequently lower level of activity for the remainder of the session.

Analysis of variance indicated significant effects of groups $(F, 3,36=8.3)$ time $(F 8,208=4.1)$ and interaction $(F 24,208=4.9 ; p<.001$ in all cases .

\section{Discussion}

A moderate dose of opiates produced a temporary state of immobility which could be briefly interrupted by a variety of arousing environmental procedures. Despite the initial behavioral arousal, the normal period of immobility was prolonged. This may speak to two distinctive aspects of the catalepsy syndrome; normal motor initiation and tonic immobility, which may be differentially affected by environmental procedures.

Two interpretations of these findings are possible. One draws upon a possible biphasic effect of stress, while the second assumes two underlying behavioral mechanisms of satalepsy. By the first explanation the apparent dissociation may be due to the fact that an asute stress may have different properties than a more prolonged stress. Interactions with acute or more prolonged stress may differ because of altered properties of stimulation. By the second interpretation two catalepsy mediating mechanisms may be differentially stress responsive, and in fact responsive in diametrically opposed manner.

In either case the present observations are of interest. The present findings suggest catalepsy may be a behaviorally complex syndrome, which is controlled by several underlying psychobiological mechanisms. They further provide evidence that stress may affect opiate syndromes and that catalepsy may be more accurately described by the term catatonia (Costall and Naylor, 1974; Papeschi et al, 1976).

\section{Acknowledgments}

The editorial assistance of Ruth Metski and Abe Feingold is gratefully acknowledged.

\section{References}

BRUNING, J. L. and KINTZ, B. L. (1977). Computational handbook of statistics. 2nd edition. Scott Foresman, Glenview, Ill.

COSTALL, B. R. and NAYLOR, R. J. (1972). On catalepsy and catatonia and the predictability of the catalepsy test for neuroleptic activity. Psychopharmacologia (Berl.) 34: 233-241.

KATZ, R. J. and CARROLL, B. J. (1978). Endocrine control of psychomotor activity. Physiol. Behav. 20: 25-30.

KRUEGER, H., EDDY, N. B. and SUMWALT, M. (1941). The pharmacology of the opium alkaloids. U.S. Govt. Public Health Service, wash., D.C., pp. 1-20.

KUCHINSKY, K. and HORNYKIEWICZ, 0 . (1972). Morphine catalepsy in the rat in relation to striatal dopamine metabolism. Eur. J. Pharmacol. 19: 119-122.

NAKUMURA, K., KUNTZMAN, A. and MAGGIO, A. H. (1973). Conney decreases in morphine's analgesic action and increase in its cataleptic action by 6-hydroxydopamine injected bilaterally into caudate and putamen areas; partial restoration by L-DOPA plus decarboxylase inhibition. Neuropharmacology 12: 1153-1160.

PAPESCHI, R., THEISS, P. and AYHAN, H. (1976). AMT catalepsy and hypokinesia; interaction with morphine and cocaine. Psychopharmacologia (Berl.) $46: 149-157$. 
Inquiries and reprint requests should be addressed to:

Dr. R.J. Katz

Mental Health Research Institute, Department of Psychiatry

The University of Michigan Medical Center, Ann Arbor, MI 48109, USA. 